\title{
MANUAL DE FORMACIÓN DE POLICÍA COMUNITARIA DE EL SALVADOR
}

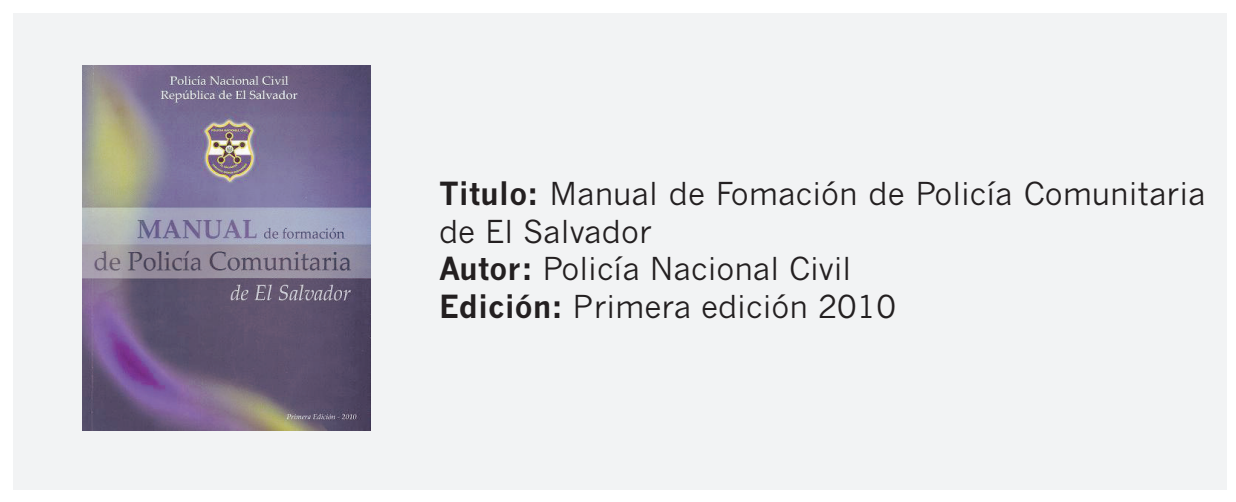

\section{Presentación}

En el mundo académico de El Salvador el papel de la institución policial, sus características, su relevancia, sus aciertos y desaciertos, y sus proyecciones siguen siendo temas pendientes de tratamiento serio y riguroso.

El Manualde Formación de Policía Comunitaria de El Salvador, no pretende llenar por completo ese vacío, sino solamente contribuir a colocar en la agenda y en la literatura nacional a la corporación policial y sus avatares. Constituye, por ende, un ejercicio de recopilación y ordenamiento de conceptos y de herramientas de trabajo en materia de Policía Comunitaria, sobre la base de la experiencia misma de la PNC, y de una bibliografía básica en esta materia.

Es un Manual de Formación con dos características fundamentales:

1. Se trata de un texto con una vocación didáctica y con una visión técnico y tecnológica; es decir, provee de herramientas de trabajo práctico, más que una revisión conceptual o teórica exhaustiva.

2. Es un Manual ligado a la línea "técnico-policial", dejando de lado lo "administrativo-organizacional".

Entendido así, con sus alcances y limitaciones, el presente Manual contiene una estructura de 11 capítulos, a saber: conceptos generales y doctrina institucional de la policía comunitaria, habilidades sociales básicas con tres ejes transversales: inserción y vinculación comunitaria, resolución de problemas, recepción de denuncias y atención ciudadana; así también elaboración de diagnósticos, Inteligencia policial, diseño de proyectos de prevención comunitaria, planificación operativa, operatividad policial, supervisión, evaluación institucional y rendición de cuentas. 
En el primer capítulo, se revisan los principales conceptos definitorios de la Filosofía de Policía Comunitaria de El Salvador y sus referentes internacionales, incluyendo sus características esenciales y sus diferencias con el modelo "tradicional" de Policía. Asimismo, se presenta la doctrina institucional que sustenta este enfoque de trabajo.

La Filosofía de Policía Comunitaria de El Salvador no es una simple réplica de alguna de las orientaciones de Policía Comunitaria ya revisadas. De una forma ecléctica, este enfoque retoma aquellos principios de trabajo que han demostrado ser útiles en la construcción e implementación de laPolicía Comunitaria en otros países, con arreglo a la realidad salvadoreña y a la propia dinámica institucional de la PNC.

De la Filosofía de Policía Comunitaria de Japón, y su aplicación por la Policía Militar de São Paulo (Koban-Bases Comunitarias de Seguridad), se ha retomado las actividades a realizar durante la operatividad policial y la identificación del rol del y la policía como movilizador de las potencialidades de la comunidad para la solución de sus problemas.

Del enfoque de Policía de Proximidad se ha retomado la ampliación del campo de acción del cuerpo policial, incluyendo temas como el bienestar de la comunidad y la mejora de su calidad de vida. Además en esta modalidad la comunidad juega un rol activo en la formulación de los objetivoslocales de seguridad que la Policía debe atender (planificación), a la vez que participa en la evaluación del logro de los objetivos (rendición de cuentas).

Del enfoque de Policía Orientada a Problemas (POP), se retoma la aplicación de una metodología policial, dividida en cuatro etapas,denominada BASE (Búsqueda, Análisis, Solución y Evaluación).

En cuanto a las habilidades sociales básicas, constituye el esfuerzo de mayor extensión con tres ejes transversales.

El propósito del primero es la introducción y ubicación adecuada de la importancia de las habilidades sociales para él y la policía en su trabajo diario, así como al planteamiento de ejercicios prácticos de tres habilidades fundamentales: "Saber escuchar, "Saber presentarse" y "Saber iniciar, mantener y cerrar una conversación".

Mientras que algunos especialistas presuponen una capacidad más o menos inherente para actuar de forma eficaz, otros sostienen que la capacidad de emitir una respuesta asertiva o socialmente hábil puede adquirirse por medio de procesos de aprendizaje.

Para determinar si una conducta es eficaz se pueden considerar tres componentes: primero, la eficacia para alcanzar los objetivos de la respuesta (eficacia en los objetivos); segundo, la eficacia para mantener o mejorar la relación con la otra persona en la interacción (eficacia en la relación), y tercero, la eficacia para mantener la autoestima de la persona socialmente habilidosa (eficacia en el respeto a uno mismo) (Caballo, 1997). 
El segundo eje está referido a la inserción comunitaria, lo cual puede en primera instancia, suponer un ejercicio innecesario para algunos, por cuanto se argumenta que la Policía, de suyo, ya es parte de la comunidad. Sin embargo, la realidad indica que, pese a que físicamente la Policía se encuentre dentro de una comunidad, no siempre establece relaciones efectivas y armoniosas con ella.

Junto con la comunidad, con arreglo a una adecuada inserción en la misma, la Policía está en grado de identificar conjuntamente factoresde riesgo e investigar los problemas desde adentro y desde su origen, así como conocer los factores sociales de protección allí presentes. Estainformación, obtenida así, sustenta de mejor forma la elaboración de diagnósticos y las posteriores propuestas de planes operativos.

Así, la inserción comunitaria tiene como propósitos específicos: Fortalecer o recuperar la confianza de la población, identificar los miembros que ejercen liderazgo en la comunidad, conocer cómo está organizada la comunidad, conocer sus problemas, amenazas, necesidades e intereses, además de conocer sus capacidades o potencialidades.

El tercer eje plantea la posibilidad que las funciones y destrezas tradicionales de la Policía no se adapten necesariamente a las nuevas modalidades de inseguridad y, por ello, surge la necesidad de ampliar conocimientos, enfoques y habilidades. La Policía Comunitaria ha demostrado, en tal sentido, mayor afinidad con métodos alternativos de trabajo, como es el caso del método de resolución de problemas.

En este capítulo se introducen, teórica y prácticamente, los principios fundamentales del método de resolución de problemas. Asimismo, mediante el análisis y los ejercicios prácticos, se favorece el aprendizaje de este método, adaptándolo a las posibilidades de la PNC y las realidades específicas del país.

\section{Recepción de denuncias y atención ciudadana}

Aunque lo parezca, captar adecuadamente una denuncia no es fácil, entre otras razones, a que las mismas se encuentran muchas veces las claves y las pistas necesarias para solucionar el caso. Por otra parte, existe la posibilidad de que en el acto de la denuncia se genere una comunicación que le permita a el y la policía obtener más información o de mayor calidad; la cual, a su vez, podrá eventualmente ser parte del ejercicio diagnóstico e insumo para la planificación de los servicios policiales.

Lo mismo puede y debe decirse del contacto ciudadano. Éste es entendido como el establecimiento de relaciones comunicativas entre el ciudadano y el personal policial, las cuales están regidas por un propósito concreto, preferiblemente definido por el o la policía.

En el Manual de Policía Comunitaria se busca dar consistencia y revalorizar el acto de la toma de denuncias y la atención ciudadana como actividades de primer orden en el quehacer policial. 


\section{Elaboración de diagnósticos}

La fase de diagnóstico de todo proceso provee de los insumos necesarios para la toma de decisiones estratégicas y operativas. Con base a uno adecuado es posible dirigir en mejor forma las acciones que habrán de seguirse.

La fase diagnóstica es, justamente, valiosa por cumplir con un doble cometido: guía de la planificación y parámetro de evaluación.

En esta parte del contenido, con apoyo en la tradición propia de la investigación social, se estudian algunos conceptos fundamentales y herramientas básicas para elaborar tres tipos de diagnósticos, a saber: diagnóstico de incidencia delictiva, diagnóstico de factores de riesgo y diagnóstico de aspectos socio. comunitarios.

\section{Inteligencia policial}

Concerniente a esta materia se pretende revisar el llamado ciclo de inteligencia, su importancia y aplicación en la Filosofía de Policía Comunitaria, entendido como un proceso de trabajo que tiene un input -diagnósticos-y un output -operatividad policial.

El y la policía que trabaja en las unidades territoriales está en la obligación de dominar un repertorio de conocimientos y destrezas amplias. Esta obligación deriva de una realidad: su ubicación en la calle como escenario de trabajo, lo expone a multiplicidad de circunstancias y situaciones.

Dicha afirmación supone tres cosas: a) Que el trabajo comunitario requiere manejo de información y centra su eficacia preventiva en captar y procesar adecuada y oportunamente información. b) Implica la posibilidad de captar información para investigar y reprimir delitos que afectan a la comunidad. c) Sin que sea una forma de manipulación de la comunidad, se debe proveer de la información necesaria para ayudar a prevenir y/o combatir el crimen.

\section{Diseño de proyectos de prevención comunitaria}

En el primer apartado de este rubro se realiza una aproximación rápida respecto al tema de la prevención comunitaria, así como al rol que, en ella, juegan la comunidad y la Policía, habida cuenta que los y las policías asumen, cada vez con mayor frecuencia, la iniciativa en el desarrollo de proyectos de corte social que buscan prevenir la delincuencia.

Los proyectos sociales, por su parte, son cada vez más complejos en cuanto a su diseño, desarrollo y evaluación. En aras de garantizar el conseguimiento de los resultados y salvaguardar la inversión, esta área de trabajo se ha hecho, con el tiempo, cada vez más tecnificada, al punto de requerir conocimientos y metodologías específicas. 


\section{Planificación operativa}

En este capítulo se analizan los principales pasos y contenidos del proceso de planificación operativa, de acuerdo a la Filosofía de Policía Comunitaria, reconociendo su importancia y valor práctico.

Las actividades policiales deben responder a una planificación previa, en la que se puedan distinguir diferentes niveles, prioridades y enfoques.

Se debe planificar la operatividad con base en, por un lado, los datos recopilados en los diagnósticos y, por otro, en la información procesada que emana de usodel ciclo de la inteligencia.

Asimismo, la planificación operativa debe ocurrir en el marco de una relación de socios con las autoridades locales y las comunidades, a fin de mejorar su calidad de vida.

\section{Operatividad policial}

La Policía como institución debe evolucionar para acompañar el desarrollo de la sociedad, diseñando políticas institucionales y estrategias orientadas a dar satisfacción a las demandas y expectativas de la comunidad.

En las policías tradicionales, el énfasis de la planificación y ejecución de la operatividad se centra en la prevención y control del delito -o reactividad frente al delito-, y como un agregado subordinado se presta atención a la prevención social y situacional, así como a las solicitudes y demandas de la comunidad.

En la Filosofía de Policía Comunitaria las prioridades cambian, atendiendo operativamente todos esos elementos de manera integral.

\section{Supervisión, evaluación institucional y rendición de cuentas}

La Filosofía de Policía Comunitaria plantea la necesidad del impulso de acciones participativas encaminadas a realizar las funciones de supervisión, evaluación institucional y rendición de cuentas.

Para efectos prácticos, se pueden distinguir tres grandes áreas de aplicación, cada una de las cuales tiene propósitos, métodos e, incluso, actores diferentes, a saber: control interno, control externo y rendición de cuentas.

La bibliografía de referencia permitió sustentar académicamente el "Manual de Formación de Policía Comunitaria de El Salvador" recurriéndose a una importante cantidad literatura nacional e internacional atinentes al área del trabajo social, la psicología, la administración pública, la gestión de calidad, las ciencias policiales, el liderazgo y a documentos institucionales propios. 